\title{
Rubber Fibre Composite Modelling and its Influence on Fatigue Damage Assessment
}

\author{
Simon Oman ${ }^{1}$, Marko Nagode ${ }^{2}$, and Jernej Klemenc ${ }^{2}$ \\ ${ }^{1}$ University of Ljubljana, Faculty of Mechanical Engineering \\ ${ }^{2}$ University of Ljubljana
}

August 11, 2020

\begin{abstract}
A novel multi-axial energy-based approach is presented and used to demonstrate the influence of different finite element (FE) modelling techniques on the prediction of the fatigue life of a rubber composite with long oriented fibres. It is shown that the simplest modelling methods using $2 \mathrm{D}$ elements with rebar layers, layered 2D elements or layered 3D elements do not allow for a precise determination of the critical location and damage value. In contrast, modelling methods with 3D matrix and discrete reinforcement provide much better results. The predicted critical location corresponds to the measured one, although the predicted fatigue life still differs from the measured results. The most complex microscopic modelling method shows the best agreement between the predicted and measured fatigue life. Since microscopic modelling is not suitable for modelling larger products made of rubber fibre composite, it is also noted that modelling techniques with 3D matrix and discrete reinforcing elements can be used with the same accuracy if the fatigue life curve is obtained from measurements on the specimens made of composite material rather than the specimens made of the critical base material (rubber).
\end{abstract}

\section{Hosted file}

Manuscript.docx available at https://authorea.com/users/349921/articles/475134-rubber-fibrecomposite-modelling-and-its-influence-on-fatigue-damage-assessment

\begin{tabular}{|c|c|c|c|c|c|}
\hline Material Model & \multicolumn{2}{|c|}{$\begin{array}{l}\text { Hyperelastic Marlow } \\
\text { (defined by uniaxial test) }\end{array}$} & Elastic & $\begin{array}{l}\text { Transversely isotropic } \\
\text { elastic }\end{array}$ & Orthotropic elastic \\
\hline Section & \multicolumn{2}{|l|}{ Rubber matrix } & $\begin{array}{l}\text { Rebar layers }+ \text { truss } \\
\text { elements }\end{array}$ & $\begin{array}{l}\text { Sections of reinforced } \\
\text { material - micro model }\end{array}$ & $\begin{array}{l}\text { Reinforcement plies } \\
\text { - mixed modelling }\end{array}$ \\
\hline \multirow[t]{10}{*}{ Parameters } & Nom. Stress [MPa] & Strain [/] & $E=2300 \mathrm{MPa}$ & $E_{1}=20 \mathrm{MPa}$ & $E_{l}=1800 \mathrm{MPa}$ \\
\hline & 0 & 0 & $v=0.45$ & $E_{2}=20 \mathrm{MPa}$ & $E_{2}=1 \mathrm{MPa}$ \\
\hline & 0.2738 & 0.1346 & & $E_{3}=2300 \mathrm{MPa}$ & $E_{3}=1 \mathrm{MPa}$ \\
\hline & 0.4467 & 0.2607 & & $v_{l 2}=0.45$ & $v_{12}=0.002$ \\
\hline & 0.5717 & 0.4004 & & $v_{13}=0.004$ & $v_{13}=0.002$ \\
\hline & 0.6721 & 0.5365 & & $v_{23}=0.004$ & $v_{23}=0.45$ \\
\hline & 0.7427 & 0.6699 & & $G_{I 2}=6.896 \mathrm{MPa}$ & $G_{I 2}=0.33 \mathrm{MPa}$ \\
\hline & 0.8150 & 0.8017 & & $G_{13}=50 \mathrm{MPa}$ & $G_{13}=0.33 \mathrm{MPa}$ \\
\hline & 0.9336 & 0.9288 & & $G_{23}=50 \mathrm{MPa}$ & $G_{23}=0.345 \mathrm{MPa}$ \\
\hline & 1.0867 & 1.0696 & & & \\
\hline
\end{tabular}




\begin{tabular}{lll}
\hline Modelling technique & $\begin{array}{l}U_{a e}^{\text {cycle }} \\
{\left[\mathrm{J} / \mathrm{mm}^{3}\right]}\end{array}$ & $\begin{array}{l}\text { Fatigue life } N \\
{[\text { cycles }]}\end{array}$ \\
\hline 2D structural matrix with rebar layers & 0.224 & $2.5 \times 10^{8}$ \\
3D matrix + embedded 2D surface elements with rebar layer & 0.294 & $5.4 \times 10^{7}$ \\
3D matrix + 2D surface elements with rebar layer & 0.334 & $2.63 \times 10^{7}$ \\
3D matrix + embedded 1D truss elements & 0.250 & $1.35 \times 10^{8}$ \\
2D mixed modelling & 0.058 & $5.11 \times 10^{11}$ \\
3D mixed modelling & 0.054 & $7.64 \times 10^{11}$ \\
Complete 3D micro modelling & 0.540 & $1.75 \times 10^{6}$ \\
\hline Measured results (average) & $/$ & $1.92 \times 10^{6}$ \\
\hline
\end{tabular}



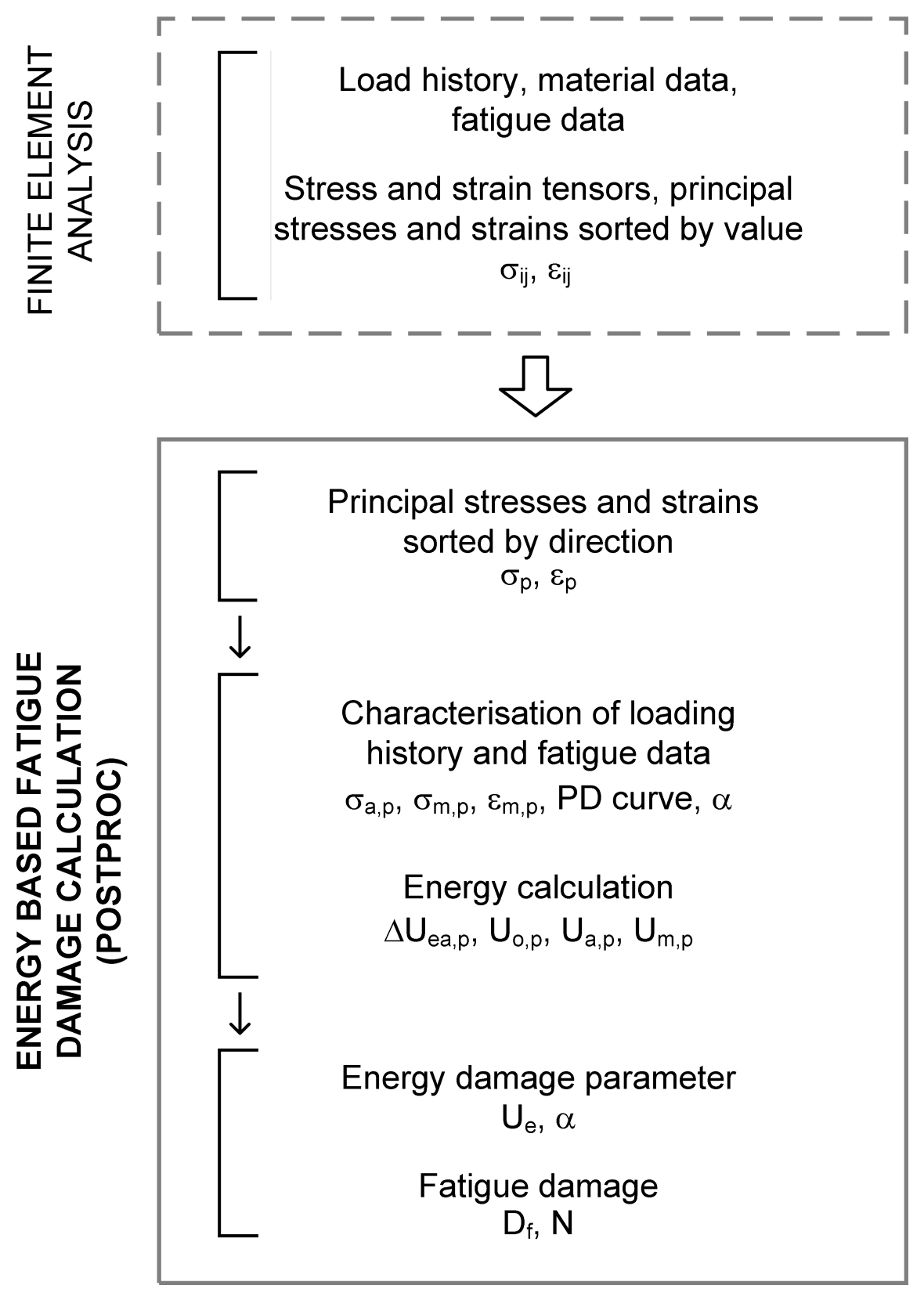

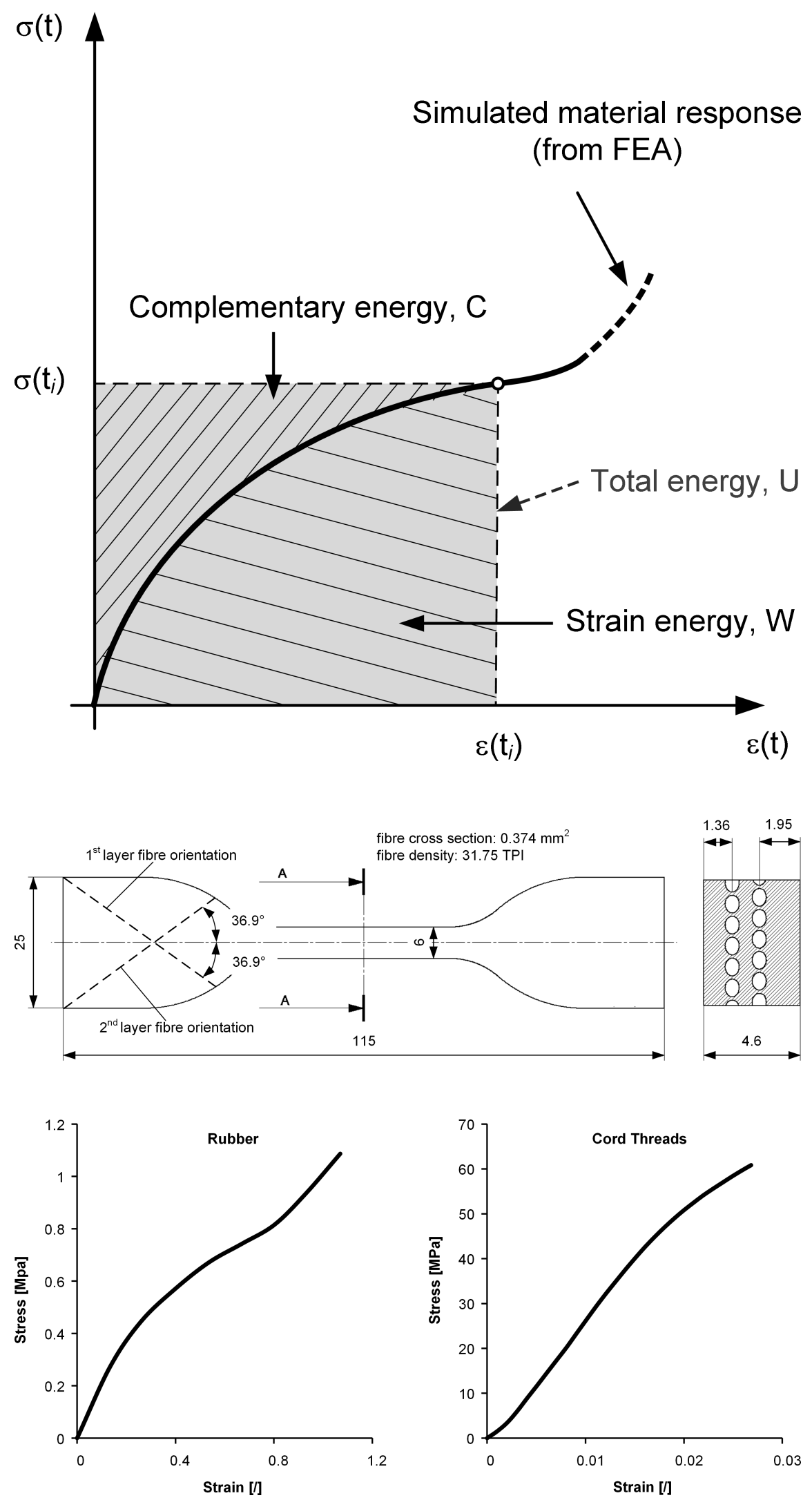

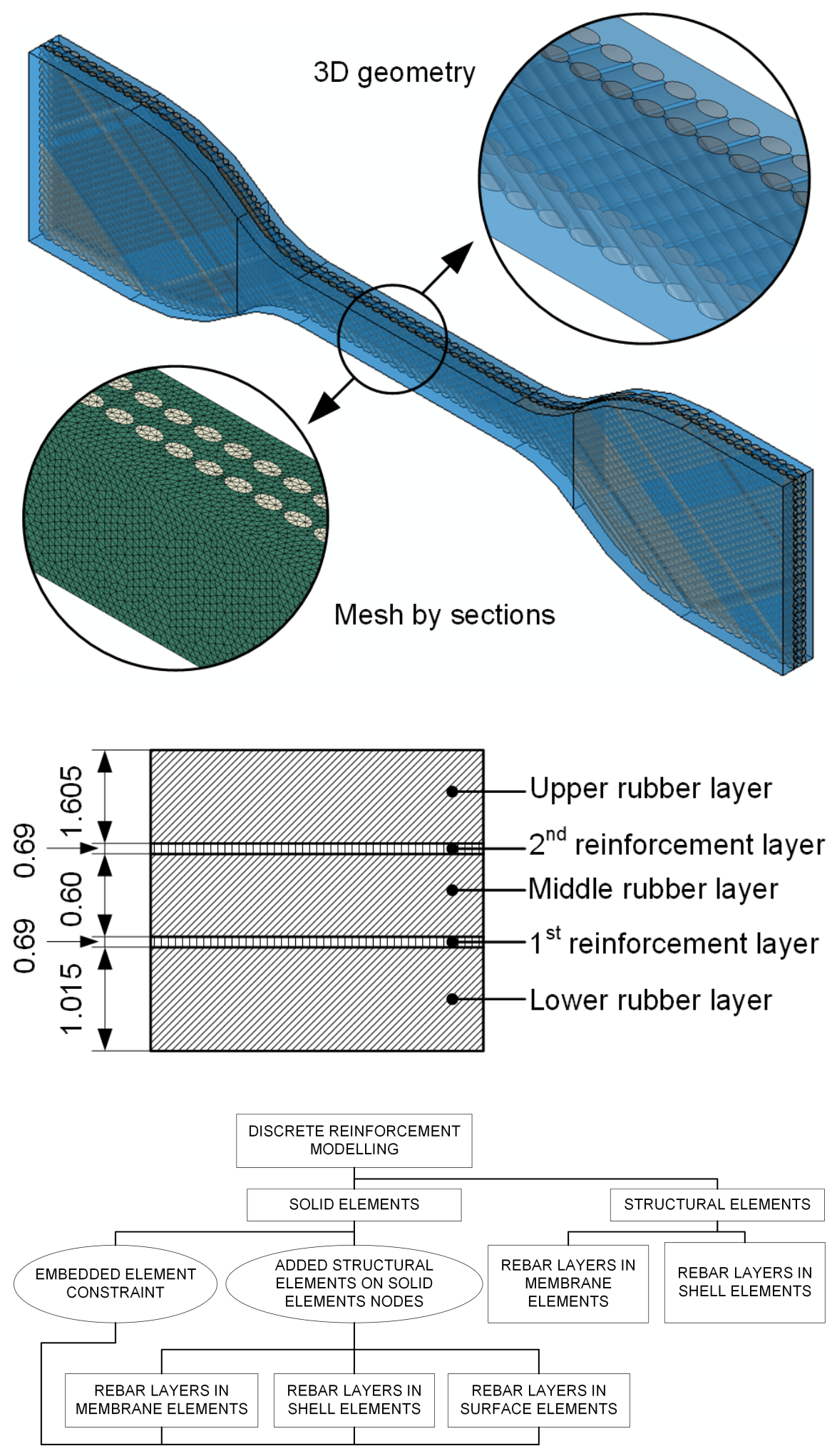

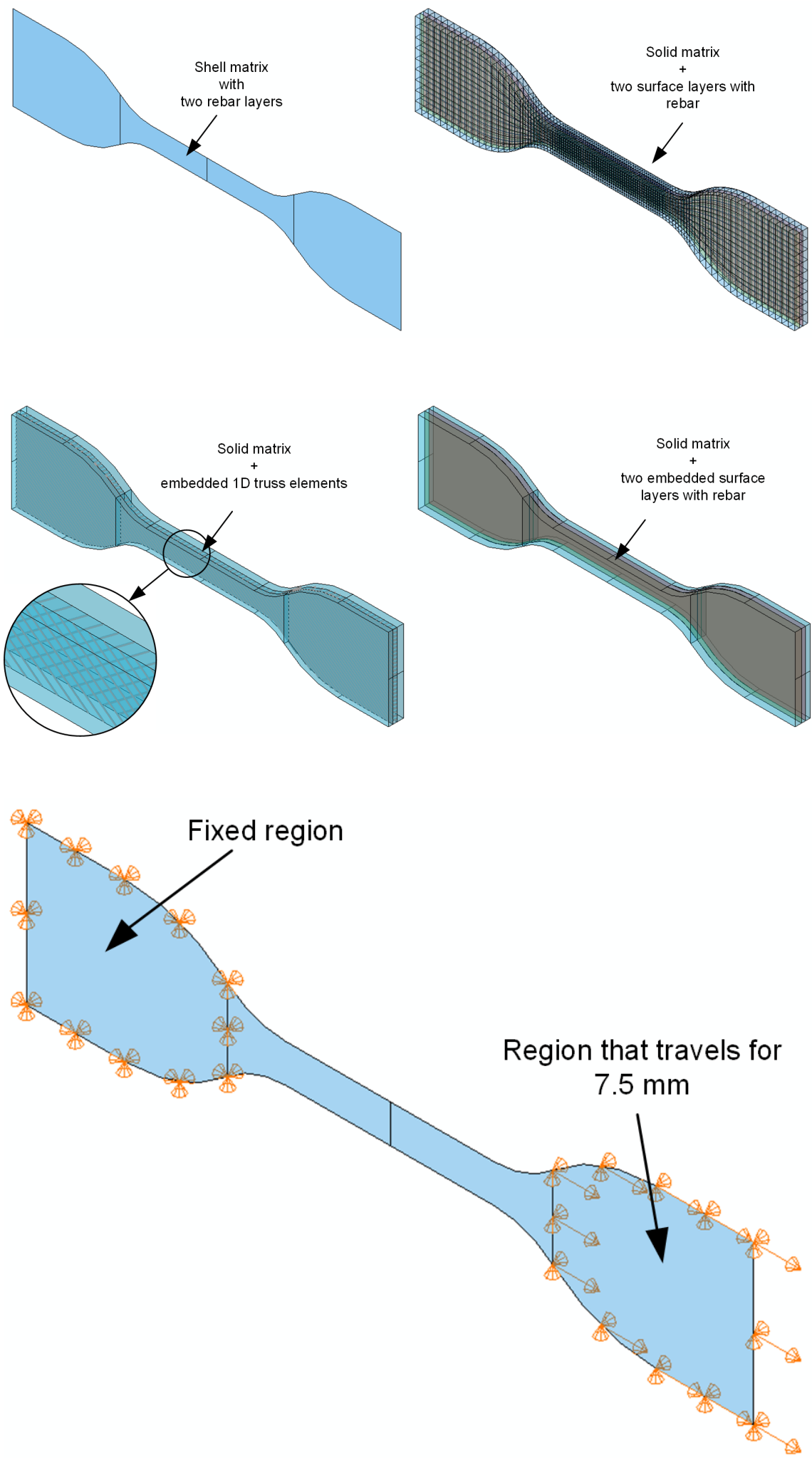


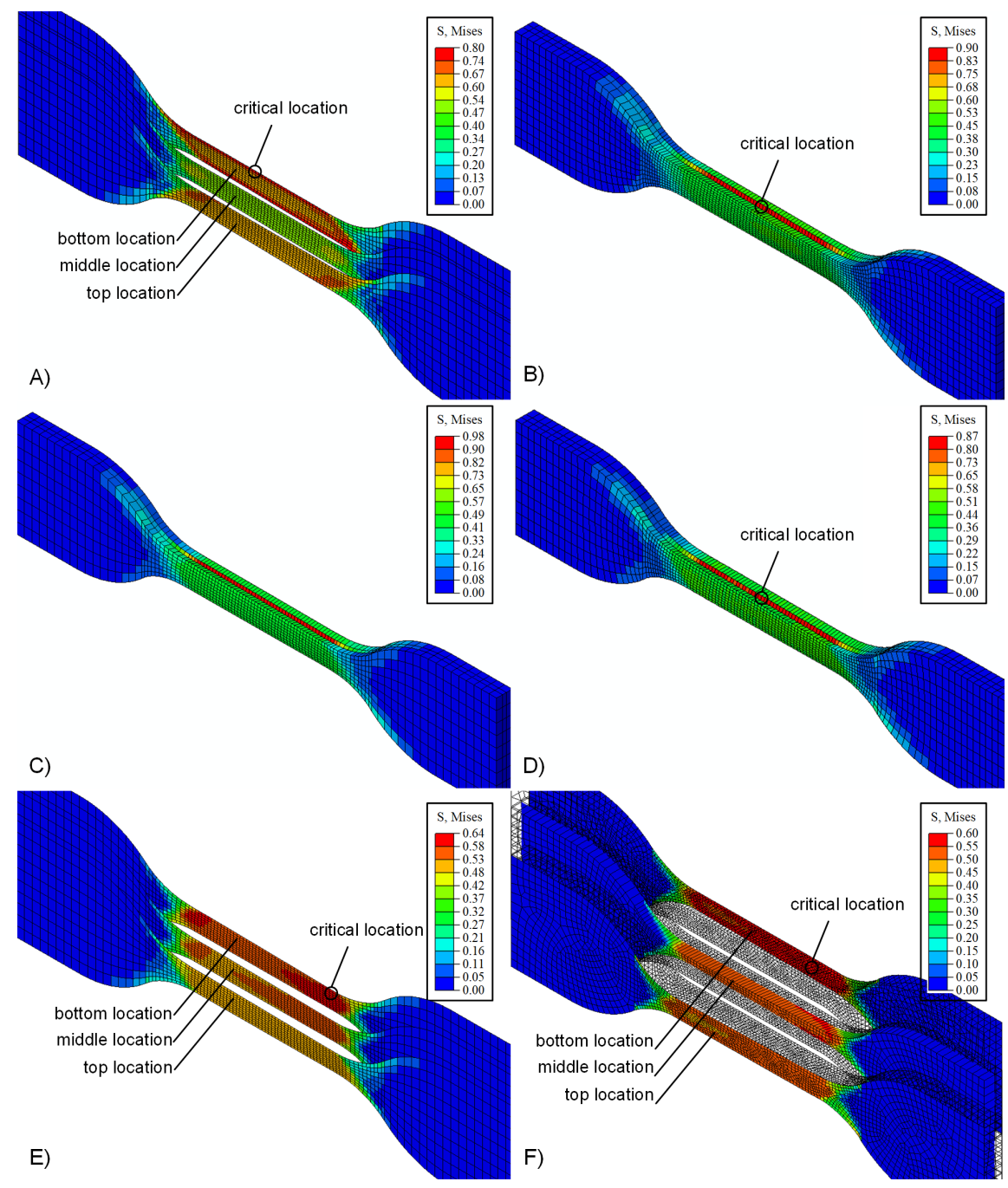




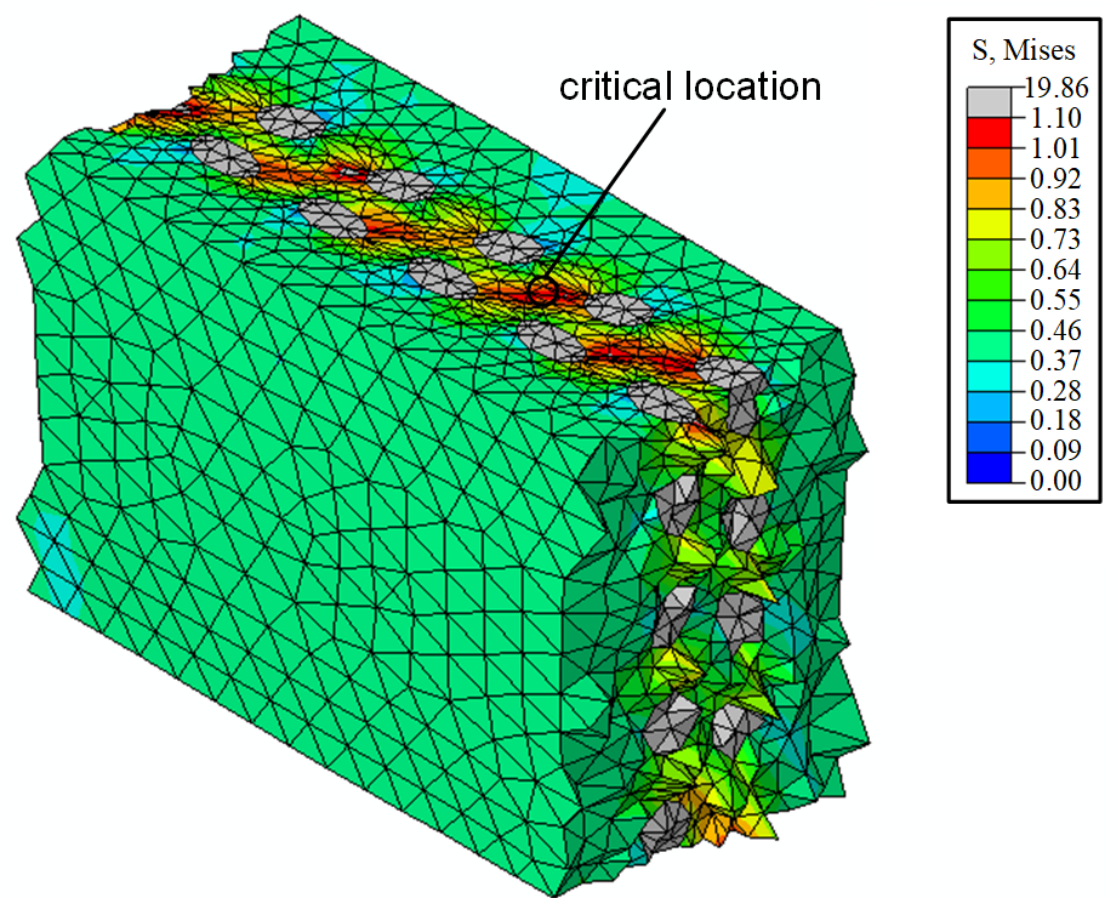




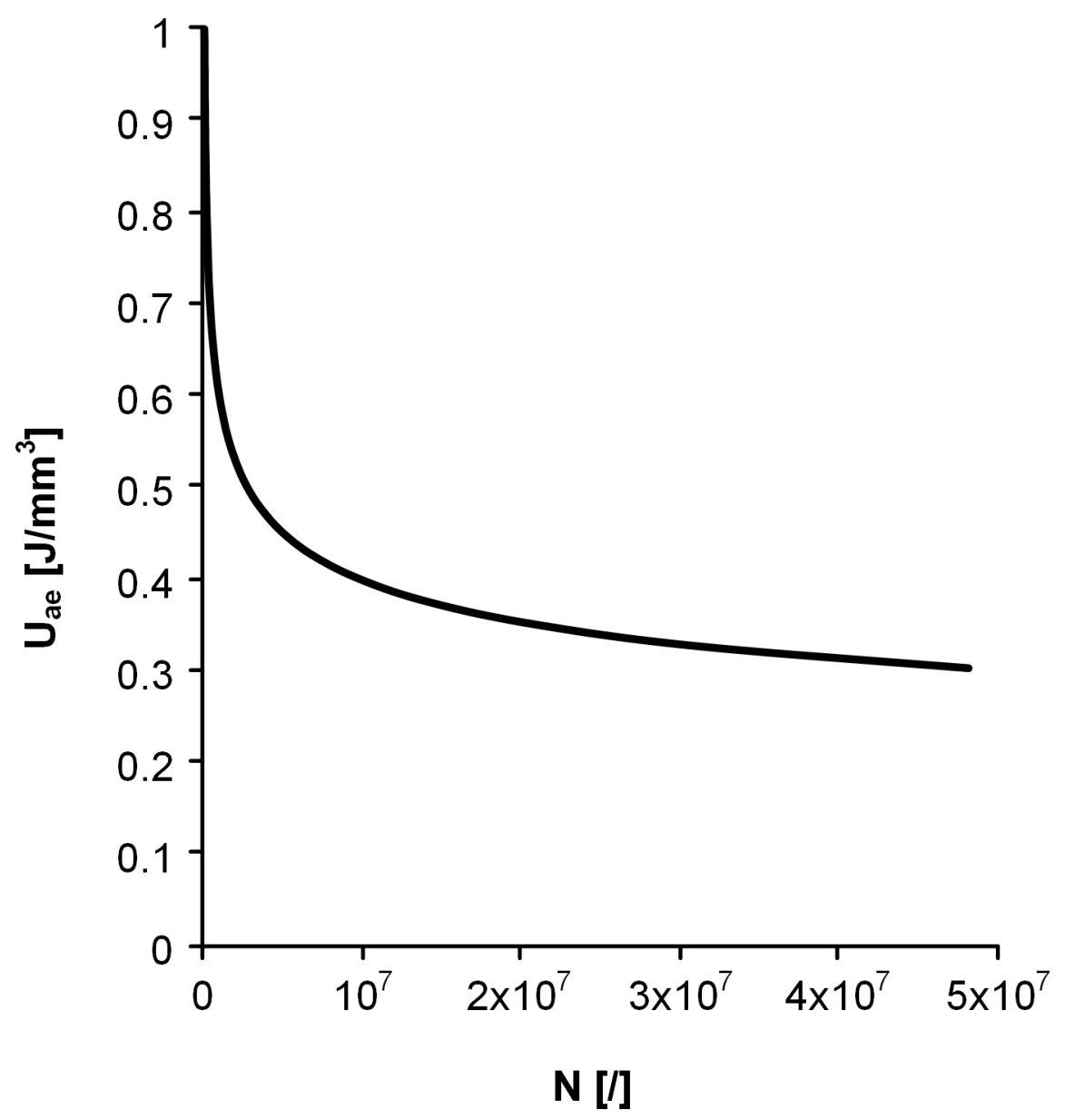

